\title{
IX Anhang
}

\section{Kommentierte Übersetzung eines Auszuges aus der Homilie des Erzbischofs Leon von Thessaloniki zu Maria Verkündigung}

Die von Vitalien Laurent 1964 veröffentlichte Homiliee $^{1}$ enthält wichtige Informationen zur Acheiropoietos-Basilika. Um einen leichteren Zugang zu diesem Schriftzeugnis zu gewährleisten, ist die relevante Passage hier in deutscher Übersetzung wiedergegeben. Die Übersetzung bezieht sich bei geringen Auslassungen auf die Zeilen 103-179 des Textes.

Es handelt sich um eine Homilie, die durch einen Codex des 12. Jhs. auf uns gekommen ist ${ }^{2}$. Sie wurde von einem gewissen Leon, Erzbischof von Thessaloniki, anlässlich des Festes der Verkündigung Marias gehalten. Die von Laurent vorgenommene Zuweisung dieser Homilie an den Bilderfeind Leo Mathematikos, der 840-843 Erzbischof von Thessaloniki war, ist ausgeschlossen, da in der Homilie von Bildern in der Kirche die Rede ist (s. u.) $)^{3}$. Außerdem ist der Name Leon verbreitet und die Liste der Bischöfe Thessalonikis nicht lückenlos überliefert ${ }^{4}$. Möglicherweise handelt es sich bei dem Autor um den Bischof dieses Namens, der um die Mitte des 12. Jhs. im Amt war ${ }^{5}$.

In dem Text bezieht sich der Redner auf ein Wunder, das zur Zeit des Kaisers Anastasios I. (491-518) in der Kirche stattgefunden haben soll und eine Massenbekehrung der Juden von Thessaloniki zur Folge hatte. Während die Identifizierung von Heiligen anhand von Bildern und die Bekehrung von Juden zum Christentum aufgrund einer wundersamen Heilung in der hagiographischen Literatur Topoi darstellen ${ }^{6}$, dienen bestimmte Details der Versachlichung des Wunders, die offenbar die Glaubwürdigkeit erhöhen sollen.

Diese Detailangaben wurden anscheinend aus einer oder mehreren älteren Quellen übernommen, die bereits in frühbyzantinischer Zeit abgefasst worden

1 Laurent 1964, 297-302.

2 LAURENT 1964, 282.

3 Zur Zuweisung der Homilie an Leon Mathematikos Laurent 1964, 282-287. Zur Person s. auch Lilie 2000, 51-54 Nr. 4440. Laurents Zuweisung wurde bereits von Kazhdan 1991, 1217 und Cormack 1969, 51 Anm. 128 aufgrund der bilderfeindlichen Einstellung Leons angezweifelt.

$4 \mathrm{Zu}$ einer neueren Bischofsliste Thessalonikis s. Fedalto 1988, 423-429.

5 So bereits Cormack 1969, Anm. 128. Zu diesem Leo s. Fedalto 1988, 426; Laurent 1963, 334 f. Nr. 460.

6 Dagron 1991, 30 f.; von Falkenhausen 2008, $24 \mathrm{f}$. 
sein könnten ${ }^{7}$. Jedenfalls sind die Angaben zur Amtszeit des Bischofs Andreas korrekt und auch die Bischöfe Eudoxos und Aristeides sind anderweitig durch zeitgenössische Quellen bezeugt ${ }^{8}$. Auch die in dem Wunder erwähnte Taufe des Basileides im Erwachsenenalter kurz vor dessen Tod ist im Mittelalter nicht mehr üblich?. Die Verwechslung des Baptisteriums mit einem öffentlichen Bad durch die Protagonistin des Wunders passt bis in die frühe mittelbyzantinische Zeit zu den örtlichen Gegebenheiten ${ }^{10}$. Die Angabe, dass Aristeides die von Kaiser Anastasius gestiftete Geldsumme später verschwendet habe ${ }^{11}$, gehört nicht zum eigentlichen Wunderbericht. Möglicherweise stammt dieses Detail wie auch die Angabe des Vorgängers und des Nachfolgers des Andreas aus einer Art Bischofsbuch der Kirche von Thessaloniki, wo ähnlich wie im Liber pontificalis in chronologischer Reihenfolge die Stiftungen und Baumaßnahmen der Bischöfe vermerkt waren ${ }^{12}$. Es bleibt jedoch unklar, ob Bischof Leo für seine Homilie eine voll ausgebildete schriftliche Fassung des Wunders verwendete, welche die genannten Detailangaben bereits enthielt.

„In der Amtszeit des heiligen Andreas aus Hierapolis am Euphrat als Vorsitzender dieser christusfreundlichen Stadt - in diesem Amt folgte er dem Eudoxos $^{13}$, nachdem er von Kaiser Zenon [474-491] zum Bischof erkoren worden war und unter Anastasios [491-518] den größten Teil seiner Amtszeit als Bischof durchlaufen hatte - ist folgendes geschehen: Ein 15jähriges jüdisches Mädchen, das von Geburt an taubstumm war [...] und beim Phallos ${ }^{14}$ wohnte (Phallos war das Dionysos Heiligtum, wo die Heiden das abscheuliche Phallosfest begingen), [...] hatte im Traum eine Vision gehabt; es sah nämlich einen

7 Ähnlich bereits Cormack 1969, $50 \mathrm{f}$ : „,Whatever its date of composition, the author evidently had access to some accurate historical source, [...]."

8 Nach Laurent 1964, 292 ist der in Z. 162 erwähnte Aristeides mit dem späteren Bischof von Thessaloniki identisch, der unter Bischof Dorotheos, dem Nachfolger des Andreas (vgl. Kap. V.1 S. 197 Anm. 12), noch als Presbyter bezeugt ist. In einem Brief des Papstes Hormisdas vom Januar 519 wird er als Presbyter erwähnt. Dazu Bolonakēs 1976, 102 f. Die Vita Davids von Thessaloniki bezeugt ihn als Bischof der Stadt um 535. $\mathrm{Zu}$ den entsprechenden Textauszügen aus der Vita Davids s. SAvvidis 1985, 314-316.

9 Z. 134 f. Der Brauch, die Taufe auf dem Sterbebett zu empfangen, wird in der Spätantike zunehmend von der Kindertaufe verdrängt. KLeijwegt - Amedick 2004, $917-$ 920; vgl. Ristow 1998, 84. Seit justinanischer Zeit war die Taufe von Neugeborenen gesetzlich vorgeschrieben (Cod. Iust. I 11, 10).

10 Z. $122-126$. s. u. Anm. 18.

11 Z. $159-163$.

12 Zum Liber Pontificalis s. die Edition von Duchesne.

13 Zum Vorgänger des Andreas und dessen Amtszeit Laurent 1964, 291 f. In Konzilsakten

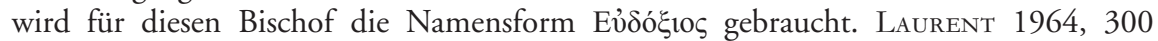
Anm. 3.

14 BaKalakēs 1985, 984-989 verortet den Dionysos-Tempel und das jüdische Viertel der frühbyzantinischen Zeit westlich der Agora. 
weißgekleideten Mann und eine sittsame Frau mit ihm, die es beide zusammen von ihrem Haus mitnahmen und entlang der breiten Straße ${ }^{15}$ bis zu diesem ehrwürdigen Haus unserer Herrin, der unbefleckten Gottesgebärerin, führten. Sie ließen das Mädchen vor dem südlichen Propylon stehen und verschwan$\operatorname{den}^{16}$.

Das Mädchen stand dort ratlos, bis es einige Menschen sah, die zur heiligen Taufe hinein schritten ${ }^{17}$; es ging auch mit ihnen hinein und traf auf einige Menschen, die sich taufen ließen. Es dachte, es sei in einem öffentlichen Balaneion [...], wo es sich samt seiner Kleider [in das Taufbecken] hineinsetzen wollte, um ein Bad zu nehmen ${ }^{18}$. Nachdem es dreimal eingetaucht war, tauchte es aus dem heiligen Wasser auf und sah den Mann und die Frau, die es diesen Weg entlang geführt hatten; sie sagten zu ihr: „Hör und sprich zu Deinem Volk“. So hat das Mädchen sofort in seinem Traum sein Gehör und seine Stimme erlangt. Da wachte es auf [...] und unbemerkt von seinen Verwandten schlich es sich aus seinem Haus und lief die Straße entlang, die ihm im Traum gezeigt worden war, erreichte diese heilige Kirche und fand das heilige Taufbecken voll mit Wasser vor, wegen der Taufe eines alten Mannes namens Basileides, der in seinen letzten Atemzügen noch die heilige Taufe empfing. Dort, wie aus göttlichem Gebot von niemandem gehindert und wie im Traum so auch im Wachsein, machte es sich frei von seinen Kleidern, sprang ins Wasser und stand mit gebeugtem Nacken voller Enthusiasmus vor dem Geistlichen, der damals die Eingeweihten [Katechumenen] taufte. Der Geistliche war der oben erwähnte Bischof Andreas. Der durch diesen Wagemut des Mädchens in Staunen versetzte Bischof begriff sehr schnell durch die göttliche Eingebung,

15 Der im Text Z. 119 gebrauchte Begriff $\lambda \varepsilon \omega \varphi o ́ \rho \circ \varsigma$ bezeichnete in byzantinischer Zeit die heutige Egnatia Straße. Laurent 1964, 293. Der Kirchenbau und das Propylon konnten wahrscheinlich ursprünglich direkt von dieser Hauptstraße aus betreten werden. So Kourkoutidou-NikolaïDou 1989, 8. 15.

16 Die Acheiropoietos-Basilika ist die einzige der Theotokos geweihte frühbyzantinische Kirche von Thessaloniki, die zudem auch ein Propylon an der Südseite aufweist. LaURENT 1964, 292-294 identifiziert deshalb im Zusammenhang mit den weiteren topographischen Angaben des Textes (Baptisterium, Zugang von $\lambda \varepsilon \omega \varphi o ́ \rho \circ \varsigma)$ die erwähnte Kirche folgerichtig als die der Panhagia Acheiropoietos. Allerdings entspricht das heutige Erscheinungsbild des Propylons einer wohl mittelbyzantinischen Umbauphase. s. o. Kap. II.2 Anm. 71.

17 Aus diesem Textzusammenhang kann man erschließen, dass das Baptisterium mit der heutigen Kapelle an der Südseite der Kirche identisch ist. So auch Bakalakēs 1985, 987.

18 Bakirtzis 1983, $320 \mathrm{f}$. 324 vermutet, dass ein Teil der Thermenanlage, auf deren Gelände die Kirche errichtet wurde, auch nach dem Bau der Basilika weiter benutzt wurde. Außerdem wurden direkt südlich der Kirche eine wohl erst in der zweiten Hälfte des 5. Jhs. errichtete Badeanlage nachgewiesen. MARKē 1999, 513 f. Zeichnung 27, die dem Grabungsbericht zufolge bis in die sdunklen Jahrhunderter bzw. die frühe mittelbyzantinische Zeit in Betrieb war. Bis zu dieser Zeit würde diese Verwechslung für einen Leser bzw. Hörer dieses Wunders in einem nachvollziehbaren Rahmen stattfinden. 
dass so etwas nicht ohne göttliche Hilfe geschehen kann, und so, ohne im geringsten zu zögern, legte er seine Hand auf den Kopf des Mädchens und taufte es, das nur im Traum die Katechese erlebt hatte. Dann stieg das Mädchen aus dem Wasser, und, [...] obwohl es vorher taubstumm war, fing es an, zu sprechen und zu hören und erzählte das, was ihm im Traum offenbart worden war. Gleichzeitig blickte es auf eines der im heiligen Baptisterium angebrachten Bilder ${ }^{19}$ mit der Darstellung von mehreren Gestalten, zeigte auf die Gestalten unserer heiligen Gottesmutter und des ruhmvollen Märtyrers Demetrios ${ }^{20}$ und sagte, dass die beiden es waren, die ihm im Traum nachts erschienen waren; indem es laut den Glauben der Christen beschwor, sagte es, dass sie es waren. Die Namen der Heiligen wußte es nicht zu nennen [...] ja wußte überhaupt nicht, ob sie Heilige seien; es hat lediglich ihre Gestalten erkannt.

19 Es ist davon auszugehen, dass der Autor auf wirklich vorhandene Bilder verweist (z. B. Ikonen), um die Glaubwürdigkeit des Wunderberichts zu erhöhen (vgl. o. Kap. IV.1.7 S. 153). Es ist nicht vollkommen auszuschließen, dass es sich dabei um einst vorhandene figürliche Darstellungen des frühbyzantinischen Mosaikschmucks handelt. Reste einer jüngeren Wanddekoration werden im Grabungsbericht von XYNGopoulos 1952a nicht erwähnt. Zu den möglicherweise einst vorhandenen Heiligendarstellungen im Baptisterium o. Kap. III.3.5 mit den Ausführungen zu Mosaik Ach34 und u. Anm. 20.

20 Der Heilige Demetrios wird weiter oben im Text (Z. 117 f.) als weißgekleidet bezeichnet. Dies erinnert an die frühchristlichen Mosaikdarstellungen des Märtyrers in der Demetrios-Basilika von Thessaloniki, wo er nahezu ausnahmslos eine weiße Chlamys und meist auch eine weiße Tunika trägt. Zur Tracht des Demetrios s. die Abb. 282-287. 289-292. 294. 297-299 sowie Tassias 2002a, 24. 27. 30. Vgl. auch das Fresko mit dem heiligen Demetrios in Santa Maria Antiqua in Rom mit weißer Tunika und gelbroter Chlamys: Nordhagen 1978, 105 f. Taf. 28-29. Die Erwähnung der weißen Kleidung ist jedoch nicht zwingend ein Reflex frühbyzantinischer Darstellungen des Heiligen (o. Anm. 19), da in der byzantinischen Hagiographie das weiße Kleid einen literarischen Topos darstellt. Vgl. Pratsch 2005, 213. Jedenfalls kann sich diese Stelle nicht auf die von Konstantinos Armenopoulos im 14. Jh. erwähnten Ikonen des heiligen Demetrios und der Gottesmutter beziehen, da der Märtyrer dort in Rüstung und mit Waffen dargestellt war. So bereits Laurent 1964, 294 Anm. 52. Zu den Ikonen XYNgOpOulos 1952b, 7-12. 20-25. Nach XyNGOpoulos kann ihre Entstehung nicht vor dem 9./10. Jh. angesetzt werden. Eine vor dem Ikonoklasmus zu datierende Darstellung des bewaffneten Demetrios ist bisher nicht nachweisbar. Vgl. Walter 2003, $77 \mathrm{f}$; Bakirtzis 1995, 60-62. Speck 1993, 342. $384 \mathrm{f}$. sieht das topische Motiv der Identifikation der Heiligen mit Hilfe von Bildern (vgl. DAGRoN 1991, $30 \mathrm{f}$. mit Beispielen) als spät an (er hält diesen Topos auch in anderen Zusammenhängen für interpoliert) und ist der Meinung, dass das geschilderte Wunder besser in die Demetrios-Kirche passe und dann erst später auf die Acheiropoietos-Basilika übertragen worden sei. Dies ist m.E. abwegig, da bei der Demetrios-Basilika die Existenz eines Baptisteriums bisher nicht sicher nachgewiesen werden konnte. Es wurde z.T. ohne belastbare archäologische Evidenz in einem Raum an der nordwestlichen Ecke der Kirche vermutet. Vgl. BoloNakēs 1976, 99 f. und Kazamia-Tsernou 2009, 273 f. Ristow 1998, 165 Nr. 296 dagegen führt dieses ‘Baptisterium` unter den „sicher oder sehr wahrscheinlich als Baptisterien zu deutenden Befunden“. 
Durch dieses Wunder sind damals viele Juden zum christlichen Glauben übergetreten; man spricht sogar davon, dass an jenem Tag der Marienverkündigung um die 500 Juden samt ihren Frauen und Kindern sich als Katechumenen eintragen ließen und am darauffolgenden Auferstehungstag die Taufe empfingen.

Als Kaiser Anastasios von diesem Wunder erfuhr, war er dermaßen begeistert, dass er unmittelbar 1000 Goldstücke an die Kirche von Thessaloniki sandte; dieses Geld, samt weiteren 800 Goldstücken, die Aristeides vorfand, verschwendete er innerhalb kurzer Zeit. Die vorher taubstumme Jüdin hat nach ihrer Heilung [...] nie diese Kirche verlassen [...] Basileides, den wir vorher erwähnt haben, starb zwei Stunden nach dem Empfang der Taufe. Durch diesen Vorfall ist ihr klar geworden, dass man im Leben keine zweite Gelegenheit erhält, um die Taufe zu empfangen, und so wählte sie das asketische Leben in Gebet und Fürbitten; man hat sie Nea Anna genannt, weil sie mit prophetischer Gabe begnadet war oder weil sie sich ständig in der Kirche aufhielt."

(Übersetzung Dr. Athanasios Alex. Fourlas) 


\section{Tabellen}

Tab. 1: Übersicht über die relative Chronologie der frühbyzantinischen Wandmosaiken von Thessaloniki

\begin{tabular}{|c|c|c|}
\hline Denkmal & Kommentar & $\begin{array}{l}\text { Datierungsgrundlage / } \\
\text { Datierungsvorschlag }\end{array}$ \\
\hline $\begin{array}{l}\text { Hagios Georgios, } \\
\text { Kuppelmosaiken, } \\
\text { Gewölbemosaiken der } \\
\text { südöstlichen und } \\
\text { westlichen Nische }\end{array}$ & $\begin{array}{l}\text { wohl wenige Jahre/ } \\
\text { Jahrzehnte jünger als die } \\
\text { Mosaiken der } \\
\text { Acheiropoietos-Basilika }\end{array}$ & $\begin{array}{l}\text { C-14-Untersuchung, } \\
\text { kunstgeschichtliche } \\
\text { Argumente / } 428 \text { - ca. } \\
\text { frühes 6. Jh. (Kap. IV.2.3) }\end{array}$ \\
\hline $\begin{array}{l}\text { Hagios Georgios, } \\
\text { Gewölbemosaik der } \\
\text { südlichen Nische }\end{array}$ & $\begin{array}{l}\text { unterschiedliche } \\
\text { Bettungsschichten und } \\
\text { größere Tesserae als die } \\
\text { restlichen Mosaiken; } \\
\text { möglicherweise jüngere } \\
\text { Phase }\end{array}$ & $\begin{array}{l}\text { Motivanalyse / erstes } \\
\text { Viertel 6. Jh. (nach SpIESER } \\
\text { 2005, 438; SpIESER 1984, } \\
\text { 135-141) }\end{array}$ \\
\hline $\begin{array}{l}\text { Mosaikfragment aus dem } \\
\text { Gebäude Egnatia Straße } \\
\text { 57/Mpakatselos Straße }\end{array}$ & $\begin{array}{l}\text { Dekor erinnert angeblich } \\
\text { an Mosaiken von Hagios } \\
\text { Georgios }\end{array}$ & $\begin{array}{l}\text { Mauerwerk des Baus als } \\
\text { Anhaltpunkt / 6. Jh. (?) } \\
\text { (Kap. IV.2.2) }\end{array}$ \\
\hline Acheiropoietos-Basilika & $\begin{array}{l}\text { wohl wenige Jahre/ } \\
\text { Jahrzehnte nach den } \\
\text { Mosaiken von Hagios } \\
\text { Georgios }\end{array}$ & $\begin{array}{l}\text { Stilanalyse / ca. 470er Jahre } \\
\text { - erstes Viertel 6. Jh. (Kap. } \\
\text { V) }\end{array}$ \\
\hline $\begin{array}{l}\text { Mosaikfragmente aus dem } \\
\text { Bau in der Demetrios } \\
\text { Gounarēs-Straße }\end{array}$ & $\begin{array}{l}\text { in zeitlicher Nähe zu den } \\
\text { Mosaiken der } \\
\text { Acheiropoietos-Basilika }\end{array}$ & $\begin{array}{l}\text { Stilanalyse / } \\
\text { fortgeschrittenes 5. Jh. - ca. } \\
\text { erstes Drittel 6. Jh. (Kap. } \\
\text { IV.2.2) }\end{array}$ \\
\hline $\begin{array}{l}\text { Mosaikfragment aus dem } \\
\text { Gebäude Iasonidou/Ecke } \\
\text { Arrianou Straße }\end{array}$ & $\begin{array}{l}\text { kunstgeschichtliche } \\
\text { Einordnung derzeit nicht } \\
\text { möglich, da nicht in Abb. } \\
\text { publiziert }\end{array}$ & $\begin{array}{l}\text { Datierung des Baus und } \\
\text { der Ausstattungsphasen } \\
\text { (Bodenmosaiken) als } \\
\text { Anhaltspunkte / 5. oder } 6 \text {. } \\
\text { Jh. (?) (Kap. IV.2.2) }\end{array}$ \\
\hline $\begin{array}{l}\text { Apsismosaik von Hosios } \\
\text { David }\end{array}$ & $\begin{array}{l}\text { wohl einige Zeit nach den } \\
\text { Mosaiken der } \\
\text { Acheiropoietos-Basilika, in } \\
\text { zeitlicher Nähe zu den } \\
\text { älteren Mosaiken von } \\
\text { Hagios Demetrios }\end{array}$ & $\begin{array}{l}\text { Stilkritische Anhaltspunkte } \\
\text { / ca. erste Hälfte 6. Jh. } \\
\text { (Kap. IV.1.6) }\end{array}$ \\
\hline $\begin{array}{l}\text { Hagios Demetrios / erste } \\
\text { Ausstattungsphase }\end{array}$ & $\begin{array}{l}\text { jünger als Mosaiken der } \\
\text { Acheiropoietos-Basilika; } \\
\text { wohl etwas jünger als das } \\
\text { Apsismosaik von Hosios } \\
\text { David }\end{array}$ & $\begin{array}{l}\text { Stilanalyse, Ikonographie, } \\
\text { epigraphische Indizien / ca. } \\
\text { zweites Drittel 6. Jh. (Kap. } \\
\text { IV.1.2; IV.1.6) }\end{array}$ \\
\hline
\end{tabular}


Tab. 1: Übersicht über die relative Chronologie der frühbyzantinischen Wandmosaiken von Thessaloniki (Fortsetzung)

\begin{tabular}{|l|l|l|}
\hline \multicolumn{1}{|c|}{ Denkmal } & \multicolumn{1}{|c|}{ Kommentar } & \multicolumn{1}{|c|}{$\begin{array}{c}\text { Datierungsgrundlage / } \\
\text { Datierungsvorschlag }\end{array}$} \\
\hline $\begin{array}{l}\text { Hagios Demetrios / zweite } \\
\text { Ausstattungsphase }\end{array}$ & $\begin{array}{l}\text { in einigem zeitlichen } \\
\text { Abstand zur ersten } \\
\text { Ausstattungsphase } \\
\text { entstanden }\end{array}$ & $\begin{array}{l}\text { historische Überlieferung, } \\
\text { epigraphische Indizien, } \\
\text { Ikonographie und } \\
\text { Stilanalyse / wahrscheinlich } \\
\text { um die Mitte des 7. Jhs. } \\
\text { (Kap. IV.1.2) }\end{array}$ \\
\hline $\begin{array}{l}\text { Mosaikfragment aus der } \\
\text { Srabung östlich der Hagia }\end{array}$ & $\begin{array}{l}\text { motivische Bezüge zu einer } \\
\text { Gemmenbordüre in } \\
\text { Hagios Demetrios }\end{array}$ & $\begin{array}{l}\text { stilistische Anhaltspunkte / } \\
\text { nicht vor dem 6. Jh., } \\
\text { möglicherweise 7. Jh. oder } \\
\text { später (Kap. IV.2.2) }\end{array}$ \\
\hline
\end{tabular}


Tab. 2: Übersicht über goldene Flächen mit schräggestellten Tesserae bei spätantiken Wandmosaiken

\begin{tabular}{|c|c|c|}
\hline Denkmal & Kontext / Literaturnachweis & Datierung \\
\hline Gerasa, Kathedrale & $\begin{array}{l}\text { Fragmente aus Grabung (Apsisbereich): } \\
\text { BRENK 1999, } 50.53 \text { f. Abb. } 1 .\end{array}$ & 5. oder 6. Jh. \\
\hline Petra, Basilika & $\begin{array}{l}\text { Fragmente aus Grabung: } \\
\text { WALISZEWSKI, in: FIEMA u.a. 2001, } \\
301 \text { WM 22. } 28 .\end{array}$ & $\begin{array}{l}\text { Mitte 5. - Mitte } \\
\text { 6. Jh. }\end{array}$ \\
\hline $\begin{array}{l}\text { Thessaloniki, } \\
\text { Acheiropoietos-Basilika }\end{array}$ & $\begin{array}{l}\text { Mosaik Ach33, Goldgrund: Kap. } \\
\text { III.3.5. }\end{array}$ & $\begin{array}{l}\text { ca. } 470 \text { er Jahre - } \\
\text { erstes Viertel } 6 . \\
\text { Jh. }\end{array}$ \\
\hline $\begin{array}{l}\text { Kartmin, Klosterkirche } \\
\text { von Mār Gabriel }\end{array}$ & $\begin{array}{l}\text { Lünettenfelder, Goldgrund: Hawkins } \\
\text { - Mundell 1973, } 289 \text { f. Abb. A-B. } \\
\text { 33-34. 40-41. }\end{array}$ & um 512 \\
\hline $\begin{array}{l}\text { Konstantinopel, } \\
\text { Polyeuktos-Kirche }\end{array}$ & $\begin{array}{l}\text { Fragmente aus Grabung: Harrison } \\
\text { 1986, 183. } 193 \text { Frag. } 98-100 \text { Abb. F. }\end{array}$ & $524-527 ?$ \\
\hline $\begin{array}{l}\text { Konstantinopel, Hagia } \\
\text { Sophia }\end{array}$ & $\begin{array}{l}\text { Wandpartien im Narthex, Goldgrund: } \\
\text { Teteriatnikov 1998, } 51 \mathrm{f.} \text {; } \\
\text { WhitTemore 1933, } 12 \text { Taf. } 3 .\end{array}$ & justinianisch \\
\hline $\begin{array}{l}\text { Lythrankomi, Panhagia } \\
\text { Kanakaria }\end{array}$ & $\begin{array}{l}\text { Fragmente aus Grabung (wohl von der } \\
\text { Apsisstirnwand): MEGAw - Hawnins } \\
1977,38 \text {. }\end{array}$ & $\begin{array}{l}\text { Erste Hälfte } 6 . \\
\text { Jh. }\end{array}$ \\
\hline $\begin{array}{l}\text { Ravenna, Sant'Agata } \\
\text { Maggiore }\end{array}$ & $\begin{array}{l}\text { Fragmente des Apsismosaiks aus } \\
\text { Grabung: Russo 1989a, } 20 \text { f. mit } \\
\text { Anm. } 21 \text { Abb. } 5 .\end{array}$ & $\begin{array}{l}\text { Erste Hälfte } 6 . \\
\text { Jh. }\end{array}$ \\
\hline $\begin{array}{l}\text { Thessaloniki, Hagios } \\
\text { Demetrios (erste } \\
\text { Ausstattungsphase) }\end{array}$ & $\begin{array}{l}\text { Nimben, Goldgrund in Mosaik Dem5: } \\
\text { Kap. IV.1.2 und IV.1.3.2. }\end{array}$ & $\begin{array}{l}\text { ca. zweites } \\
\text { Drittel 6. Jh. }\end{array}$ \\
\hline Classe, Sant'Apollinare & $\begin{array}{l}\text { Panele der Erzengel, Goldgrund: } \\
\text { Маzzotті } 1954,178 \mathrm{f} \text {. }\end{array}$ & um 549 \\
\hline Poreč, Euphrasius-Basilika & $\begin{array}{l}\text { Verschiedene kleinere Bereiche: Terry } \\
\text { - Maguire 2007, } 92 \text { Abb. 193; } \\
\text { Bernardi 2005, } 47 .\end{array}$ & Mitte 6. Jh. \\
\hline $\begin{array}{l}\text { Sinai, Kirche des } \\
\text { Katharinen-Klosters }\end{array}$ & $\begin{array}{l}\text { Stirnwandmosaiken, Goldgrund: } \\
\text { ForsyTH - WeITZMANN 1973, } 16 \\
\text { Taf. } 126-128.175 \text { a. }\end{array}$ & um 565 \\
\hline
\end{tabular}

